\title{
Review Article \\ Surface Patterning of PEDOT:PSS by Photolithography for Organic Electronic Devices
}

\author{
Shihong Ouyang, Yingtao Xie, Dongping Wang, Dalong Zhu, Xin Xu, \\ Te Tan, and Hon Hang Fong
}

National Engineering Lab for TFT-LCD Materials and Technologies, Department of Electronic Engineering, Shanghai Jiao Tong University, Shanghai 200240, China

Correspondence should be addressed to Hon Hang Fong; hhfong@sjtu.edu.cn

Received 25 July 2014; Accepted 23 December 2014

Academic Editor: Teen-Hang Meen

Copyright (C) 2015 Shihong Ouyang et al. This is an open access article distributed under the Creative Commons Attribution License, which permits unrestricted use, distribution, and reproduction in any medium, provided the original work is properly cited.

\begin{abstract}
Along with the development of organic electronics, conductive polymer of PEDOT:PSS has been attracting more and more attention because they possess various novel electrical, optical, and mechanical properties, which render them useful in modern organic optoelectronic devices. Due to its organic nature, it is lightweight and can be fabricated into flexible devices. For better device processing and integrating, it is essential to tune their surface morphologies, and photolithography is the best choice at present. In this paper, current PEDOT:PSS patterning approaches using photolithography are reviewed, and some of our works are also briefly introduced. Appropriate photolithographic patterning process for PEDOT:PSS will enable its application in future organic electronics.
\end{abstract}

\section{Introduction}

Organic electronics is a fast developing branch of modern science and technology. It has received enormous attention as a technology platform that enables lightweight, inexpensive, mechanically flexible and large-scale devices by exploiting the unique properties of organic materials. Devices such as organic light emitting diodes (OLEDs) [1], organic thin film transistors (OTFTs) [2,3], organic solar cell $[4,5]$, and sensors $[6,7]$ have been demonstrated, and tremendous progress in the performance was realized through the past two decades.

Along with the development of organic electronics, commercially available poly(3,4-ethylenedioxythiophene): poly(styrenesulfonate) (PEDOT:PSS) is emerging as a promising material for its electrical, optical, and mechanical property [8]. PEDOT:PSS is a flexible, transparent, and intrinsically conductive polymer which can reach a high conductivity above $1000 \mathrm{~S} / \mathrm{cm}$ and has a high transmission above $90 \%$ in visible spectrum $[9,10]$. It is also biocompatible and has high chemical stability. As the commonly used indium tin oxide (ITO) is brittle and will lose its conductivity during bending, PEDOT:PSS is much suitable for flexible applications. Compared with other materials like carbon nanotubes, graphene, silver nanowires, PEDOT:PSS is lowcost and can be easily deposited onto various substrates via spin-coating or printing. Moreover, PEDOT:PSS has a high work function of about $5.2 \mathrm{eV}$, which is beneficial for charge injection [1]. Due to its property, PEDOT:PSS found various applications and exhibited good performance in organic electronics including OLEDs $[1,11,12]$, OTFT $[2,3]$, OPV [4], batteries and bioelectronics $[6,7]$, and so forth.

For practical applications, it is essential to develop general patterning procedures for integrating PEDOT:PSS into microscale devices. However, micropatterning and processing of organic materials for electronic and optoelectronic systems remain a challenging issue to be addressed. Organic materials, including small molecular and polymer that can be dissolved in water or organic solvents, are usually mechanically soft and chemically sensitive. Their surface morphology and electrical property will be adversely influenced during the patterning processes. Thus, it has stimulated numerous attempts and approaches in different micro- and nanopatterning techniques for making patterned structures with fine features. Ink-jet printing involves a modified inkjet or bubble jet printer that delivers small droplets of PEDOT:PSS to selected places on the substrate [13]. It is a directly writing 
approach without any mask, and its material utilization is very high. However, it has certain resolution limitations (10$20 \mu \mathrm{m})$ as well as problems with resulting film uniformity. For imprint technique [14], it can achieve extremely high resolution possibilities $(\sim 10 \mathrm{~nm})$ using a nanostructured mold. However, due to its complexity, imprint technique still remains on the research laboratory scale level. Furthermore, for the two aforementioned methods, fabrication of multilayer devices is exceptionally challenging, and they require the use of new equipment. Photolithography, in contrast, remains the most attractive thin film patterning technique for the patterning of inorganic electronic materials to date in modern silicon-based semiconductor industry. It uses UV radiation to change the solubility of light-sensitive imaging materials in certain solutions, and the patterned imaging materials are used as a contact mask to pattern the electronic materials. However, the techniques used in the mature and entrenched industry of silicon processing have made little impact in patterning of PEDOT:PSS which is mainly due to a lack of chemical compatibility. Overcoming these incompatibilities promises a breakthrough in the application of PEDOT:PSS since it would provide for massively parallel output along with process knowledge and equipment already available from a very successful silicon industry.

In this paper, photolithographic patterning approaches of PEDOT:PSS are reviewed, and our relevant works are also briefly introduced. Using appropriate materials and processes, fine PEDOT:PSS patterns are achieved by photolithography and are applied into various organic electronics devices. These results indicate the promising applications of PEDOT:PSS in future organic electronics devices.

\section{Traditional Photolithography for PEDOT:PSS Patterning}

Photolithography has many advantages, including ultrahigh resolution ability, straightforward scaling to large area substrates, availability of a broad basis of equipment and expertise, and the high throughput associated with its inherently parallel nature. As photolithography is widely used, it will be favorable to still use materials and facilities of traditional photolithography to pattern organic material of PEDOT:PSS, especially for cost concern from industry. In spite of its technical advantages, conventional photolithography has not been recognized as a suitable technique for patterning PEDOT:PSS. It is presently hindered by concerns of chemical deterioration upon exposure to process materials for photolithography. Specifically, PEDOT:PSS films are damaged during the photoresist deposition, development, and removal steps due to interaction with aggressive chemical reagents, especially the aqueous alkaline solutions, which are standard developers in conventional photolithography. Moreover, due to the acidity of PEDOT:PSS films, the cross-linking of traditional acid-sensitive photoresists is adversely affected. For positive-tone photoresist, acidic PEDOT:PSS films cause uncontrolled decomposition. For negative-tone photoresist, the resist films are cross-linked without UV radiation, and residuals are left on the surface. Accordingly, various practical photolithographic approaches have been demonstrated to minimize or eliminate damage by careful choice of processes and relevant materials. These efforts include the utilization of physical lift-off processes and the employment of protective interlayers between PEDOT:PSS and photoresist films during etching.

In lift-off processes, shown in Figures 1(a)-1(e), photoresist is first spin-coated and patterned on a substrate, and PEDOT:PSS to be patterned are subsequently deposited on it. Appropriate solvents are used to dissolve parts of photoresist, removing PEDOT:PSS film that was deposited on it. By coating PEDOT:PSS on patterned photoresist and then performing lift-off, exposure of UV radiation and alkaline developer was prohibited, and there was also less contamination on the surface, which will be very important for ensuring good performance of electronics devices. Chan et al. used positive photoresist to performance this processes [15] and achieved micrometer-scale patterns. As positive photoresist is usually acidic sensitive and PEDOT:PSS is acidic, there is a risk that photoresist is decomposed by PEDOT:PSS solution during its spin coating. Processes like hard baking of the resist patterns may be utilized to prevent the decomposition.

Leem et al. and Huang et al. used negative photoresist SU8 $[16,17]$, as shown in Figure 2. PEDOT:PSS can be spincoated either before or after UV exposure, corresponding to route 1 and route 2 in Figure 2. Using developer of propylene glycol monomethyl ether acetate (PGMEA), unexposed parts were removed, leaving PEDOT:PSS patterns. As it is very hard to remove UV exposed SU8, cross-linked resists were left beneath PEDOT:PSS. For devices with complex structures, this will be inconvenient.

For aforementioned processes, solvents were used to remove parts of photoresist, and this may impact PEDOT:PSS films. Therefore, Takamatsu et al. and Defranco et al. performed a dry lift-off process with parylene [18, 19], as shown in Figure 3. PEDOT:PSS was spin-coated on patterned parylene, and parylene films were mechanically delaminated, avoiding the contact of solvents with PEDOT:PSS. Parylene is a chemical vapor deposited (CVD) polymer and nearly inert (resist solvents, strong acids and bases), which is useful as a barrier layer in electronic devices. It is deposited near room temperature and can be applied on chemically sensitive materials without damaging them. Most importantly, parylene has conformal and pinhole-free coating, and it shows relatively weak adhesion to substrate, which makes it easily peeled off.

For lift-off of inorganic materials, the undercut profile at the edges of patterned photoresist will ensure a discontinuous film, which results in successful lift-off, as shown in Figures 4(a) and 4(b). For a spin-coated PEDOT:PSS, however, the film is generally continuous across the edges of the patterned photoresist, shown in Figure 4(c), and this will make a rough edge of PEDOT:PSS film after peeling-off. Moreover, there may be sharp peaks at the edge, shown in Figure 4(d), which may greatly influence the device performance.

In traditional etching processes for inorganic materials, photoresist is spin-coated on the film to be patterned. After exposure and developing, photoresist patterns are formed and used as a contact mask for later etching. Finally, 


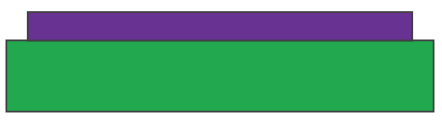

(a)
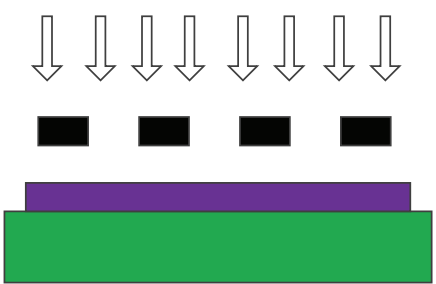

Substrate PEDOT:PSS

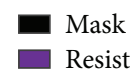

(b)

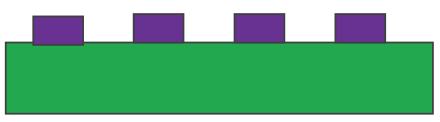

(c)

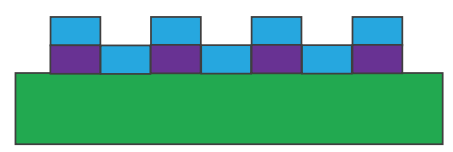

(d)

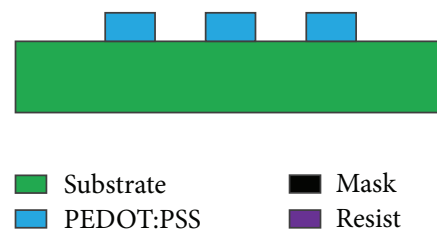

(e)

FIGURE 1: Lift-off processes of photolithographic patterning of PEDOT:PSS. Photoresist was first spin-coated (a) and patterned (b), (c) on a substrate. Then, PEDOT:PSS was subsequently deposited (d). After lift-off of left resist, PEDOT:PSS patterns are left on the substrate (e).

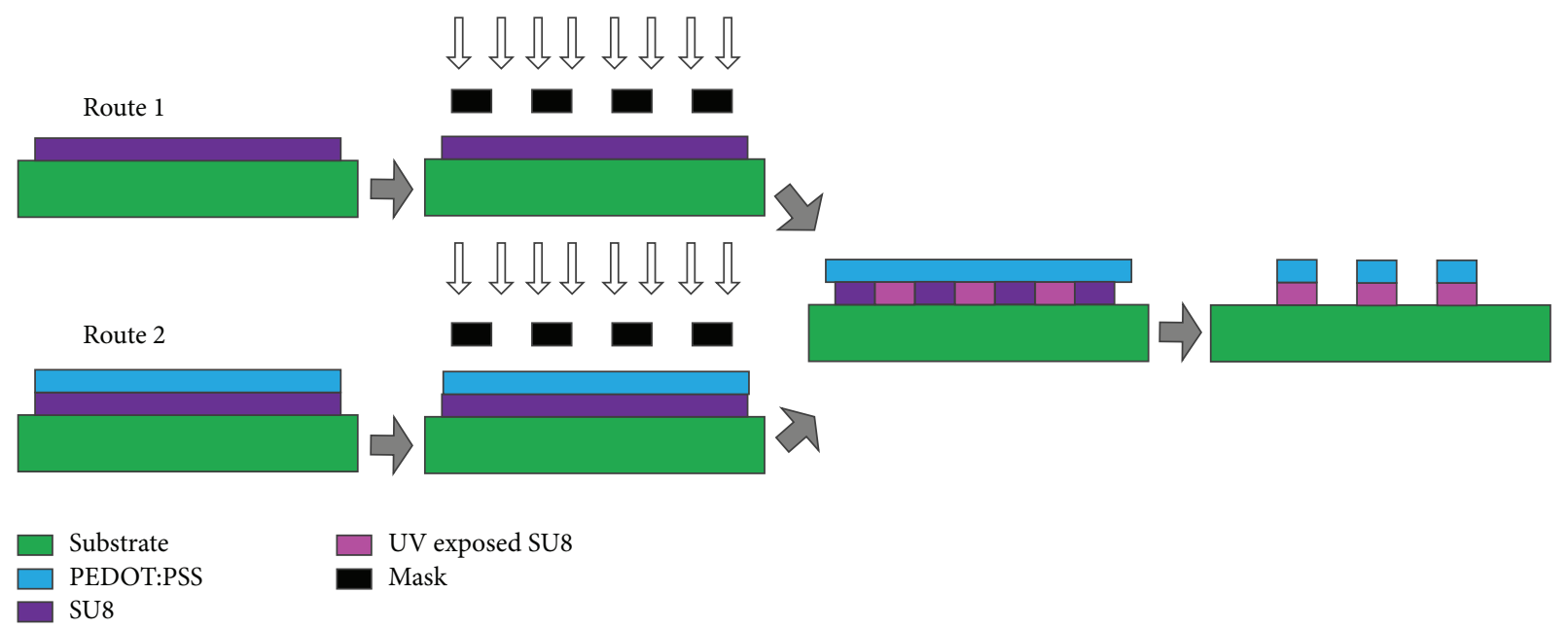

FIGURE 2: Photolithographic patterning of PEDOT:PSS using SU8. Here are two routes, in which PEDOT:PSS can be deposited either before or after UV exposure. After developing in PGMEA, PEDOT:PSS patterns are left on cross-linked SU8.

the photoresist is stripped off. For acidic PEDOT:PSS, the solvents used during the processes (especially the alkaline developer) usually have detrimental effect. Khodagholy et al. and Defranco et al. used a parylene film to protect PEDOT:PSS films $[6,19]$, as shown in Figure 5, and $1 \mu \mathrm{m}$ PEDOT:PSS lines were demonstrated. Parylene shows relatively weak adhesion to PEDOT:PSS, which makes it easily peeled off after etching. Although the conductivity of PEDOT:PSS films was proved to be unchanged during the processes, there is a risk for the surface of PEDOT:PSS to get damaged while peeling off.

As published by Xia et al., the conductivity of PEDOT:PSS can be enhanced using strong acid [10]. It is concluded that acidic PEDOT:PSS possesses strong stability in acid solution. Thus, we proposed an approach using acid as processing solvent [20]. Silver thin films were used as protective layer, and traditional photolithographic etching of PEDOT:PSS can be achieved. Shown in Figure 6, resist was first formed on silver. Using acidic silver etchant, silver interlayer was etched, followed by oxygen plasma etching of PEDOT:PSS. After resist striping and silver removal, PEDOT:PSS patterns were formed on substrate. Commonly used silver and traditional photoresist can be utilized, and this process was proved to have no obvious influence towards PEDOT:PSS.

Using processes in Figure 6, patterns down to several micrometers were achieved on a 4 -inch PET substrate (Figure 7(a)), and notably the resolution limit here is the photo mask and exposure system. With the patterned conductive PEDOT:PSS as electrode, OLEDs showed comparable performance as those with ITO anode (Figure 7(b)). 


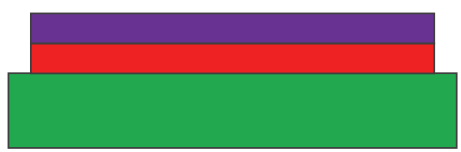

(a)
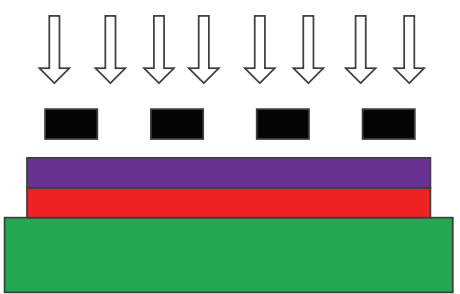

(b)

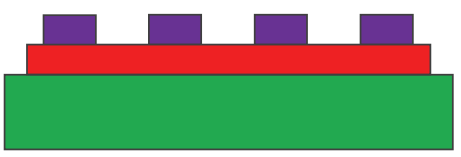

Substrate

Parylene PEDOT:PSS

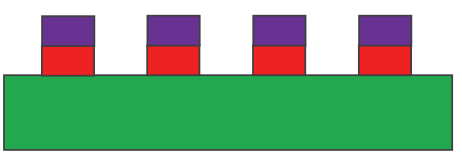

(d)

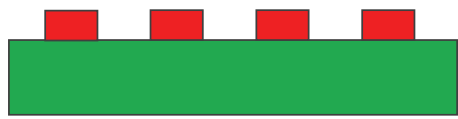

(e)

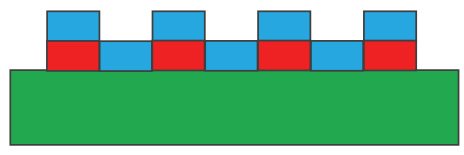

(f)

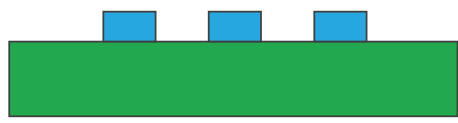

Substrate

Parylene

PEDOT:PSS

(c)

(g)

FIGURE 3: Lift-off processes with parylene. Resist was spin-coated (a), UV exposed (b), and developed (c) on parylene. With etching (d), patterns were transferred to parylene (e). PEDOT:PSS was deposited on patterned parylene (f), and after mechanical peel-off, PEDOT:PSS patterns were left on substrate $(\mathrm{g})$.

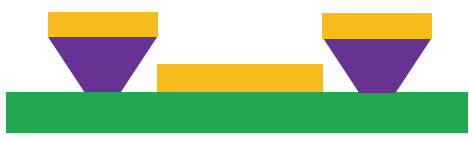

(a)

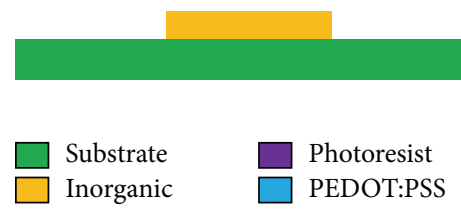

(b)

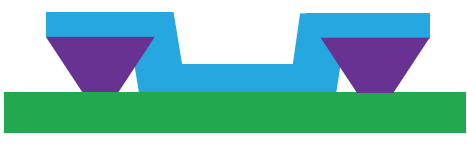

(c)

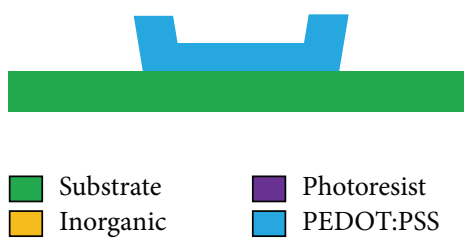

(d)

FIGURE 4: Lift-off processes of inorganic material and PEDOT:PSS. (a) Inorganic materials on photoresist, (b) inorganic material patterns on substrate after photoresist removal, (c) PEDOT:PSS on photoresist, and (d) PEDOT:PSS patterns on substrate.

\section{Orthogonal Solvents and Specially Tailored Photo Imaging Materials for PEDOT:PSS Patterning}

For photolithography in inorganic semiconductor industry, the concept of chemical orthogonality has long been practiced, and it is necessary to deposit and remove a layer of photoresist without damaging the underlying layers. However, deterioration of PEDOT:PSS occurs during the photoresist deposition and removal stages due to aggressive organic solvents, especially in the pattern development steps by aqueous base solutions. It is a major challenge in chemical processing of PEDOT:PSS films to find orthogonal solvents, that is, solvents that do not dissolve or adversely damage PEDOT:PSS. Here, two kinds of such solvents are introduced, supercritical carbon dioxide $\left(\mathrm{scCO}_{2}\right)$ and fluorous solvents.

$\mathrm{ScCO}_{2}$ is a kind of fluid which exists above the critical temperature and pressure of $\mathrm{CO}_{2}\left(T_{\mathrm{c}}=31.1^{\circ} \mathrm{C} ; P_{\mathrm{c}}=\right.$ 73.8 bar). This $\mathrm{scCO}_{2}$ fluid is a poor solvent for most ionic, high molecular weight and low vapor pressure organic 


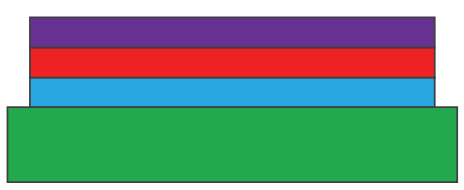

(a)
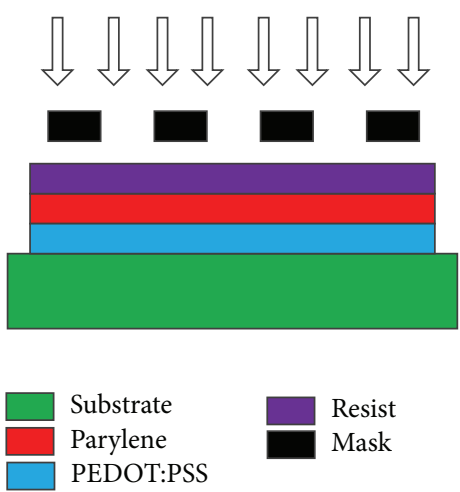

(b)

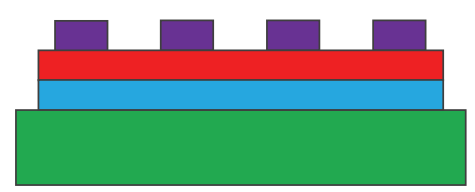

(c)

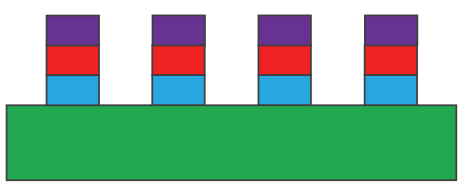

(d)
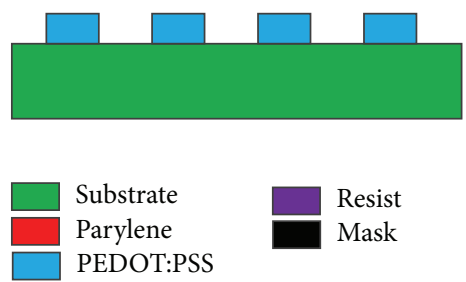

(e)

FIGURE 5: Etching process with parylene for PEDOT:PSS. (a) Spin-coating of PEDOT:PSS, parylene and resist, (b) UV exposure, (c) resist developing, (d) dry etching, and (e) parylene peeling-off.

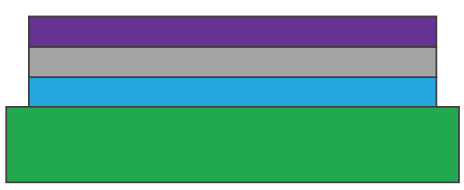

(a)
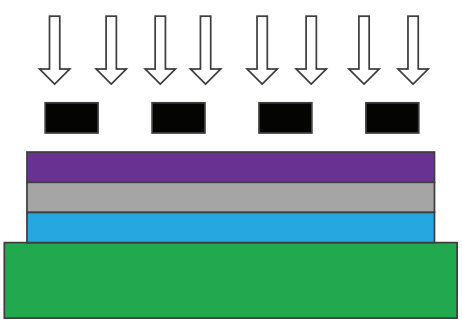

(b)

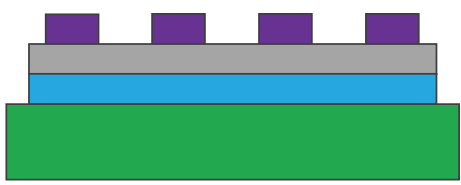

Substrate

Silver

PEDOT:PSS

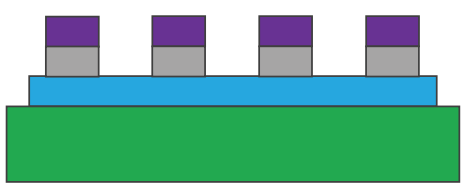

(d)

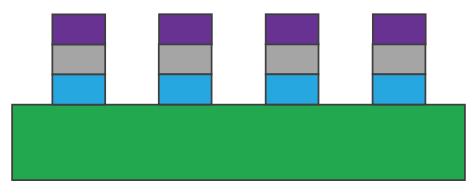

(e)
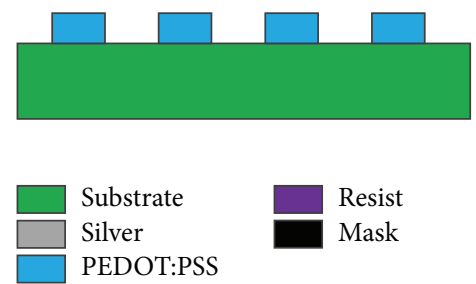

(f)

FIGURE 6: Photolithography with silver interlayer for PEDOT:PSS patterning. (a) Deposition of PEDOT:PSS, silver film and parylene. (b) UV exposure, (c) developing of resist. (d) Silver etching with silver etchant. (e) Oxygen plasma etching of PEDOT:PSS, (f) removal of resist and silver. 


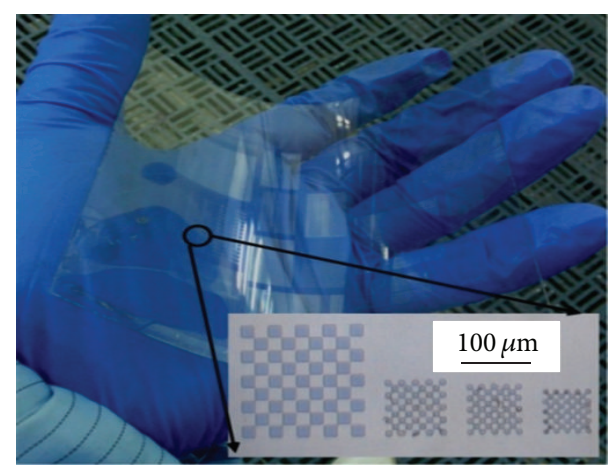

(a)

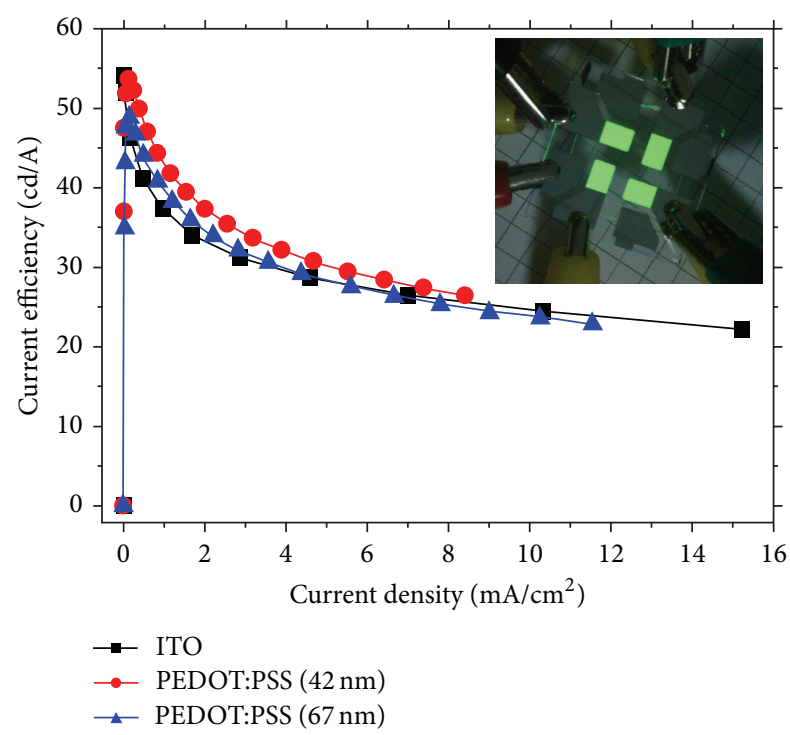

(b)

FIGURE 7: Photolithographic patterning of PEDOT:PSS using silver as protective interlayer. (a) Small patterns down to micrometer scale were fabricated on flexible substrate. (b) OLEDs using the patterned conductive PEDOT:PSS anodes had comparable performance as those using ITO anode.

compounds, which makes it an extremely promising nondestructive developing medium for vast majority of materials utilized in organic electronics. Another very important aspect is that $\mathrm{scCO}_{2}$ is a nontoxic, nonflammable, environmentally friendly fluid, which is proposed as a key driver in the new field of "green" chemistry. In recent years, $\mathrm{scCO}_{2}$ is being considered as a low-cost substitute solvent in microelectronics processing such as wafer cleaning, spin coating, etching, and resist stripping, based on its physical and chemical advantages. Utilizing dry $\mathrm{scCO}_{2}$ media as a developing solvent to dissolve resists in photolithography, Hwang et al. and Lee et al. introduced a chemically benign dry photolithographic approach without interfering with the active material, and appropriate copolymers with high fluorine content were developed (shown in Figure 8) as effective negative photoresists for development with $\mathrm{scCO}_{2}[21,22]$. Patterned PEDOT:PSS is demonstrated with fine features as small as $1.3 \mu \mathrm{m}$. No delamination, distortion, or cracking of the PEDOT:PSS patterns was observed over larger scale.

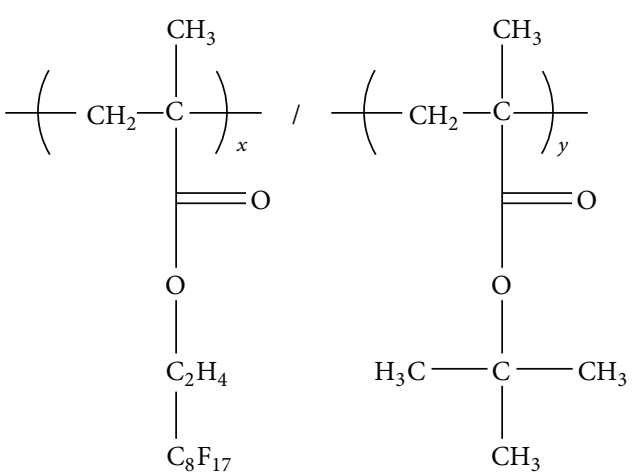

FIGURE 8: Chemical structure of photoresist for $\mathrm{scCO}_{2}$.

Fluorous solvents are perfluorinated or very highly fluorinated liquids, which are typically immiscible with organic solvents and water. They are poor solvents for nonfluorinated organic materials and benign to a wide variety of organic electronic materials, and only materials with higher F content dissolve well in fluorous solvents. Therefore, they naturally extend options for solvent orthogonality. Since hydrofluoroethers (HFEs) are well known to be highly environmentally friendly, "green" solvents, they were chosen out of the variety of other available fluorous solvents. HFEs are nonflammable, have zero ozone-depletion potential, low global warming potential, and show very low toxicity to humans. Typical commercially available HFEs are HFE 7100 (Figure 9(a)) and HFE 7500 (Figure 9(b)). Based on the HFEs solvents combined and specifically tailored, highly fluorinated photoresist (Figures 9(c) and 9(d)), Ober et al. demonstrated facile photolithographic patterning for organic electronics [23-25]. Highly fluorinated chemicals are used, including the specifically designed resist material, the resist developer solvent, and the resist stripper solvent. Any possible damage to the organic semiconductor film associated with processing the photoresist is minimized. It should be emphasized that the photosensitive resist was carefully selected to enable nonchemically amplified patterning: an imaging mechanism that does not rely on acid-catalyzed deprotection reactions. The advantages of this pathway are substantial for the patterning of acidic PEDOT:PSS films. No residual layers of decomposed resist were found, as with chemically amplified resists. The PEDOT:PSS interface is left clean and unaffected following resist removal. After photolithography processing, PEDOT:PSS films showed essentially the same resistance as the reference devices, which indicates that the photoresist has no detrimental effects on device performance. Optical and atomic force microscopy on PEDOT:PSS were also performed, before and after immersion in boiling HFE 7100. No significant change of morphology and no pinhole formation, cracking, or delamination were observed, confirming the orthogonality of HFEs even under extreme conditions. Submicrometer patterning of PEDOT:PSS was also demonstrated.

Using fluorinated provided by Orthogonal Inc., we systematically studied the influence of the patterning processes towards PEDOT:SS [26]. Both the conductivity and surface 


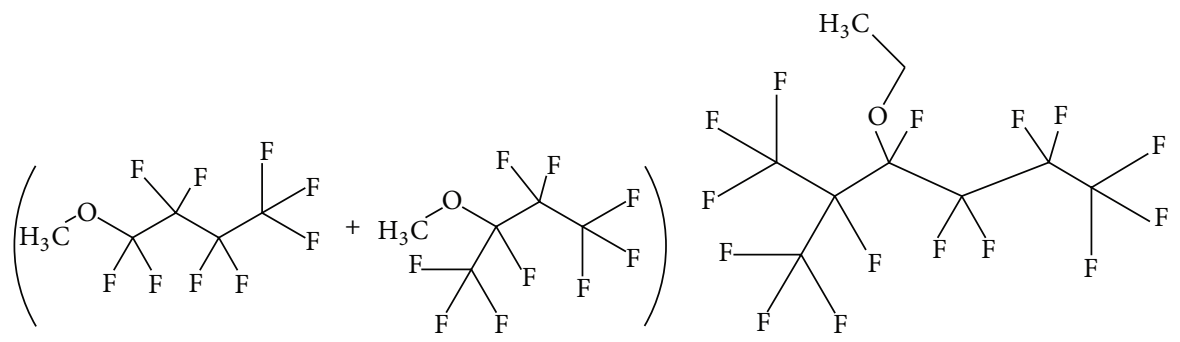

(a)

(b)

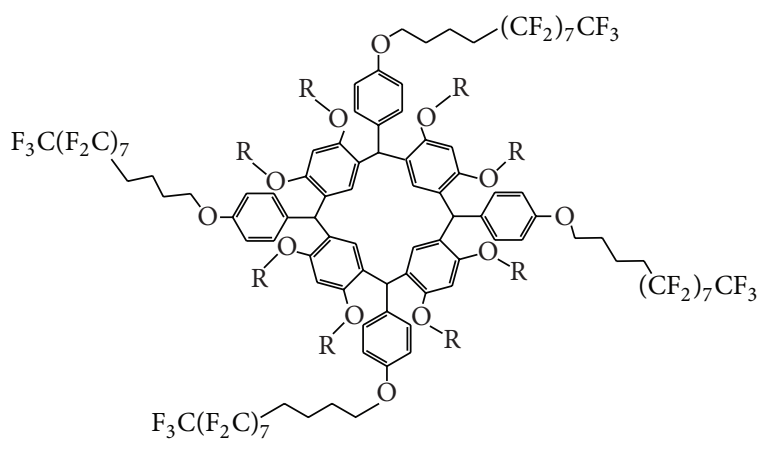

(c)

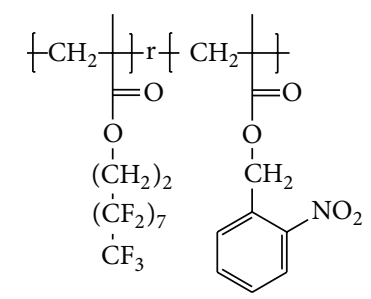

(d)

Figure 9: Different fluorinated materials. (a) HFE7100, (b) HFE7500. (c), (d) Specially designed photoresist for HFEs solvents.

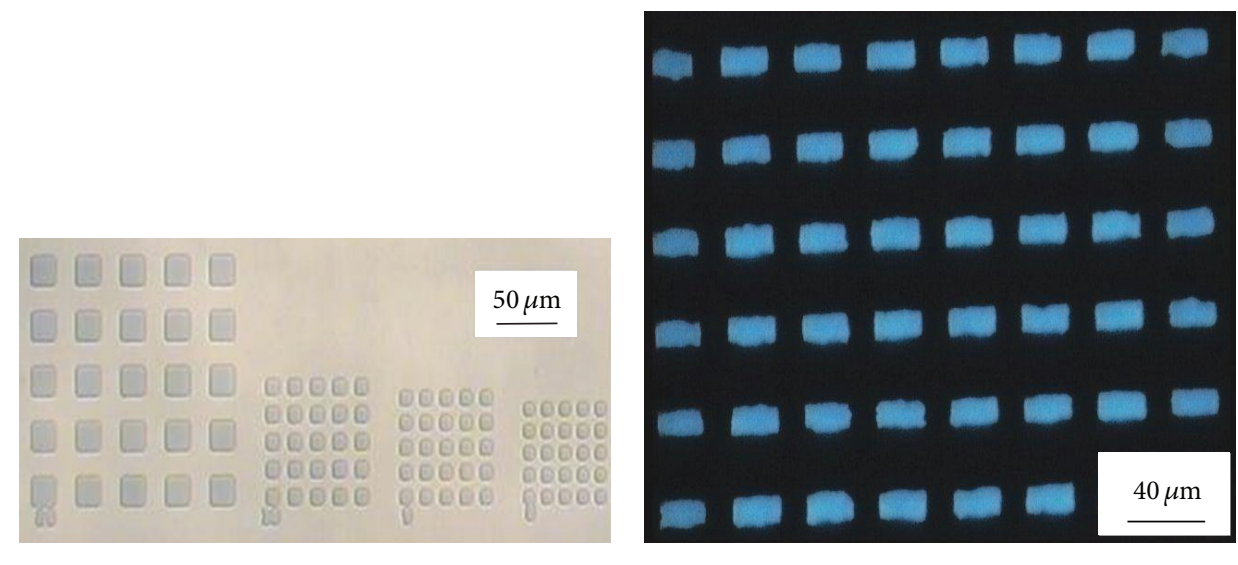

(a)

(b)

FIGURE 10: (a) PEDOT:PSS patterns by photolithography using fluorinated materials. (b) OLEDs array using patterned PEDOT:PSS strips.

property of PEDOT:PSS remained the same after patterning using fluorinated materials. Micrometer patterns were realized, shown in Figure 10(a), and the resolution here was limited by photo mask and exposure system. Based on the patterned conductive PEDOT:PSS strips, efficient OLEDs array were demonstrated, shown in Figure 10(b).

\section{Direct Cross-Linking of PEDOT:PSS}

Direct patterning by cross-linking of PEDOT:PSS has the added benefit of convenience. Through extending the well known bisfluorinated(phenyl azide) (bisFPA) methodology to an ionic bisFPA process, photocross-linking of
PEDOT:PSS is realized. This method is based on the photoinduced formation of singlet nitrenes from FPAs and their insertion into unactivated $\mathrm{C}-\mathrm{H}$ or other bonds, causing bonding of adjacent polymer chains, which is thus general and not dependent on the presence of specific chemical functional groups. However, the nitrene can also react with oxygen, which may lead to a decrease in the photosensitivity when exposed in air. The nonilluminated parts are removed using demineralized water or methanol, thus yielding a negative process. The photoinitiator should be compatible with the strongly acidic PEDOT:PSS (PH of about 1). Well-suited photoinitiators are bisazides, polyazides, or polydiazonium salts. Khong et al. demonstrated this approach and used patterned PEDOT:PSS in OLED and transistors [27-30]. 


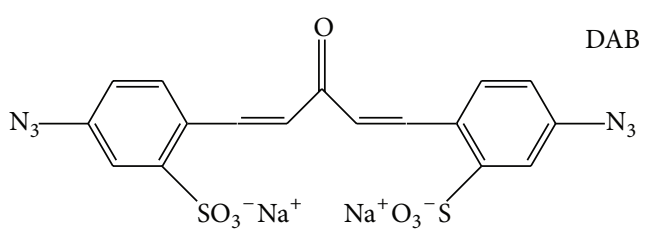

(a)<smiles>C[N+](C)(CCCNC(=O)c1c(F)c(F)c([N])c(F)c1F)CCCNC(=O)c1c(F)c(F)c(N)c(F)c1F</smiles>

(b)

FIGURE 11: Photoinitiator for direct cross-linking of PEDOT:PSS. (a) DAB, (b) AAATf.

They used water-soluble ionic photoinitiators of $4,4^{\prime}$-diazido$2,2^{\prime}$-disulfonic acid benzalacetone disodium salt (DAB) and bis(fluorinated phenyl azide) (AAATf), shown in Figures 11(a) and 11(b). The minimum feature size of PEDOT:PSS patterns realized comprises $2.5 \mu \mathrm{m}$ wide lines separated by $1 \mu \mathrm{m}$ spacing. Atomic force microscope (AFM) images of the cross-linked PEDOT:PSS films showed no significant difference in surface roughness compared to the non-cross-linked films. However, their thickness is reduced, corresponding to a retention factor of $0.7-0.8$. Conductivity enhancement was also observed after PEDOT:PSS cross-linking which could be due to the chemical doping. The cross-linking was also found to cause a work function deviation (about $0.1 \mathrm{eV}$ to $0.2 \mathrm{eV}$ ) from the original PEDOT:PSS films, and the mechanism is still not fully understood. This approach is promising, and to improve the performance of this direct cross-linking approach of PEDOT:PSS, there needs to be more research involving both materials and processes.

\section{Discussion}

For photolithographic patterning of PEDOT:PSS, different approaches can be adopted, as reviewed in above sections. For lift-off processes using conventional resist, it is convenient to obtain resists and related solvents. However, as PEDOT:PSS films are usually continuous at the edge of resist, it influences the resolution and pattern fineness. Although limit resolution also happens to lift-off with parylene, peeling-off of parylene prevents the contact of PEDOT:PSS with stripping solvents, which results in unchanged PEDOT:PSS film. However, the peeling-off processes add a little complexity. For etching with parylene as a protective layer, it can achieve very good resolution except for a little complexity by the peeling-off processes. Changing parylene with $\mathrm{Ag}$ films as a protective layer during etching, it can achieve both good resolution and simplicity, for Ag can be easily removed by acid solution, and PEDOT:PSS is acid-stable. Photolithography using orthogonal solvents and specially tailored imaging materials realized submicron PEDOT:PSS patterns, and this approach is believed to be very promising, for it can be directly transferred to conventional photolithography facilities, only to change processing materials. If adding proper photoinitiators, PEDOT:PSS can be directly cross-linked which is very convenient. However, the resolution is limited to several micrometers, and more researches on materials and processing are needed.

\section{Conclusion}

Through properly selecting materials and processes, PEDOT:PSS can be patterned using photolithography processes. With lift-off processes, traditional photolithography materials and processes can be used. If protected by interlayer, PEDOT:PSS can be patterned using traditional photolithography. Utilizing orthogonal solvents and corresponding photoresist, nanoscale PEDOT:PSS patterns are realized. PEDOT:PSS can also be directly cross-linked by UV radiation if adding proper photoinitiators. To integrated PEDOT:PSS into future organic electronic devices, however, more researches concerning both materials and processes are needed for the patterning of PEDOT:PSS.

\section{Conflict of Interests}

The authors declare that there is no conflict of interests regarding the publication of this paper.

\section{References}

[1] M. Cai, Z. Ye, T. Xiao et al., "Extremely efficient indiumtin-oxide-free green phosphorescent organic light-emitting diodes," Advanced Materials, vol. 24, no. 31, pp. 4337-4342, 2012.

[2] K. Hong, S. H. Kim, C. Yang et al., "Photopatternable, highly conductive and low work function polymer electrodes for high-performance n-type bottom contact organic transistors," Organic Electronics, vol. 12, no. 3, pp. 516-519, 2011.

[3] P. A. Ersman, D. Nilsson, J. Kawahara, G. Gustafsson, and M. Berggren, "Fast-switching all-printed organic electrochemical transistors," Organic Electronics, vol. 14, no. 5, pp. 1276-1280, 2013.

[4] S.-I. Na, S.-S. Kim, J. Jo, and D.-Y. Kim, "Efficient and flexible ITO-free organic solar cells using highly conductive polymer anodes," Advanced Materials, vol. 20, no. 21, pp. 4061-4067, 2008.

[5] M. C. Barr, C. Carbonera, R. Po, V. Bulović, and K. K. Gleason, "Cathode buffer layers based on vacuum and solution deposited poly(3,4-ethylenedioxythiophene) for efficient inverted organic solar cells," Applied Physics Letters, vol. 100, no. 18, Article ID 183301, 2012.

[6] D. Khodagholy, T. Doublet, M. Gurfinkel et al., "Highly conformable conducting polymer electrodes for in vivo recordings," Advanced Materials, vol. 23, no. 36, pp. H268-H272, 2011.

[7] D. Khodagholy, T. Doublet, P. Quilichini et al., "In vivo recordings of brain activity using organic transistors," Nature Communications, vol. 4, article 1575, 2013. 
[8] A. Elschner, S. Kirchmeyer, W. Lövenich, U. Merker, and K. Reuter, PEDOT: Principles and Applications of an Intrinsically Conductive Polymer, Taylor \& Francis, New York, NY, USA, 2010.

[9] N. Kim, S. Kee, S. H. Lee et al., "Highly conductive PEDOT:PSS nanofibrils induced by solution-processed crystallization," Advanced Materials, vol. 26, no. 14, pp. 2268-2272, 2014.

[10] Y. Xia, K. Sun, and J. Ouyang, "Solution-processed metallic conducting polymer films as transparent electrode of optoelectronic devices," Advanced Materials, vol. 24, no. 18, pp. 24362440, 2012.

[11] W. Gaynor, S. Hofmann, M. G. Christoforo et al., "Color in the corners: ITO-free white OLEDs with angular color stability," Advanced Materials, vol. 25, no. 29, pp. 4006-4013, 2013.

[12] Y. H. Kim, J. Lee, S. Hofmann, M. C. Gather, L. MüllerMeskamp, and K. Leo, "Achieving high efficiency and improved stability in ITO-free transparent organic light-emitting diodes with conductive polymer electrodes," Advanced Functional Materials, vol. 23, no. 30, pp. 3763-3769, 2013.

[13] Z. Xiong and C. Liu, "Optimization of inkjet printed PEDOT:PSS thin films through annealing processes," Organic Electronics, vol. 13, no. 9, pp. 1532-1540, 2012.

[14] H. J. Lee, T. H. Park, J. H. Choi et al., "Negative mold transfer patterned conductive polymer electrode for flexible organic light-emitting diodes," Organic Electronics, vol. 14, no. 1, pp. 416422, 2013.

[15] J. R. Chan, X. Q. Huang, and A. M. Song, "Nondestructive photolithography of conducting polymer structures," Journal of Applied Physics, vol. 99, no. 2, Article ID 023710, 2006.

[16] D.-S. Leem, P. H. Wöbkenberg, J. Huang, T. D. Anthopoulos, D. D. C. Bradley, and J. C. de Mello, "Micron-scale patterning of high conductivity poly(3,4-ethylendioxythiophene):poly (styrenesulfonate) for organic field-effect transistors," Organic Electronics, vol. 11, no. 7, pp. 1307-1312, 2010.

[17] J. Huang, R. Xia, Y. Kim et al., "Patterning of organic devices by interlayer lithography," Journal of Materials Chemistry, vol. 17, no. 11, pp. 1043-1049, 2007.

[18] S. Takamatsu, T. Takahata, K. Matsumoto, and I. Shimoyama, "Micro-patterning of a conductive polymer and an insulation polymer using the Parylene lift-off method for electrochromic displays," Journal of Micromechanics and Microengineering, vol. 21, no. 7, Article ID 075021, 2011.

[19] J. A. Defranco, B. S. Schmidt, M. Lipson, and G. G. Malliaras, "Photolithographic patterning of organic electronic materials," Organic Electronics: Physics, Materials, Applications, vol. 7, no. 1, pp. 22-28, 2006.

[20] S. Ouyang, Y. Xie, D. Zhu et al., "Photolithographic patterning of PEDOT:PSS with a silver interlayer and its application in organic light emitting diodes," Organic Electronics, vol. 15, pp. 1822-1827, 2014.

[21] H. S. Hwang, A. A. Zakhidov, J.-K. Lee et al., "Dry photolithographic patterning process for organic electronic devices using supercritical carbon dioxide as a solvent," Journal of Materials Chemistry, vol. 18, no. 26, pp. 3087-3090, 2008.

[22] J. K. Lee, P. G. Taylor, A. A. Zakhidov et al., "Orthogonal processing: a novel photolithographic patterning method for organic electronics," Journal of Photopolymer Science and Technology, vol. 22, no. 5, pp. 565-569, 2009.

[23] J. K. Lee, M. Chatzichristidi, A. A. Zakhidov et al., "Acidsensitive semiperfluoroalkyl resorcinarene: an imaging material for organic electronics," The Journal of the American Chemical Society, vol. 130, no. 35, pp. 11564-11565, 2008.
[24] P. G. Taylor, J.-K. Lee, A. A. Zakhidov et al., "Orthogonal patterning of PEDOT:PSS for organic electronics using hydrofluoroether solvents," Advanced Materials, vol. 21, no. 22, pp. 2314-2317, 2009.

[25] A. A. Zakhidov, J.-K. Lee, J. A. DeFranco et al., "Orthogonal processing: a new strategy for organic electronics," Chemical Science, vol. 2, no. 6, pp. 1178-1182, 2011.

[26] S. Ouyang, Y. Xie, D. Wang et al., "Photolithographic patterning of highly conductive PEDOT:PSS and its application in organic light-emitting diodes," Journal of Polymer Science Part B: Polymer Physics, vol. 52, no. 18, pp. 1221-1226, 2014.

[27] S.-H. Khong, S. Sivaramakrishnan, R.-Q. Png et al., "General photo-patterning of polyelectrolyte thin films via efficient ionic bis(fluorinated phenyl azide) photo-crosslinkers and their postdeposition modification," Advanced Functional Materials, vol. 17, no. 14, pp. 2490-2499, 2007.

[28] F. J. Touwslager, N. P. Willard, and D. M. de Leeuw, "I-line lithography of poly-(3,4-ethylenedioxythiophene) electrodes and application in all-polymer integrated circuits," Applied Physics Letters, vol. 81, no. 24, pp. 4556-4558, 2002.

[29] O. Fenwick, K. Oliver, and F. Cacialli, "Cross-linking of a poly(3,4-ethylene dioxythiophene):(polystyrene sulfonic acid) hole injection layer with a bis-azide salt and the effect of atmospheric processing conditions on device properties," Applied Physics Letters, vol. 100, no. 5, Article ID 053309, 2012.

[30] G. Winroth, G. Latini, D. Credgington et al., "Polyfluorenebased light-emitting diodes with an azide photocross-linked poly(3,4-ethylene dioxythiophene):(polystyrene sulfonic acid) hole-injecting layer," Applied Physics Letters, vol. 92, no. 10, Article ID 103308, 2008. 

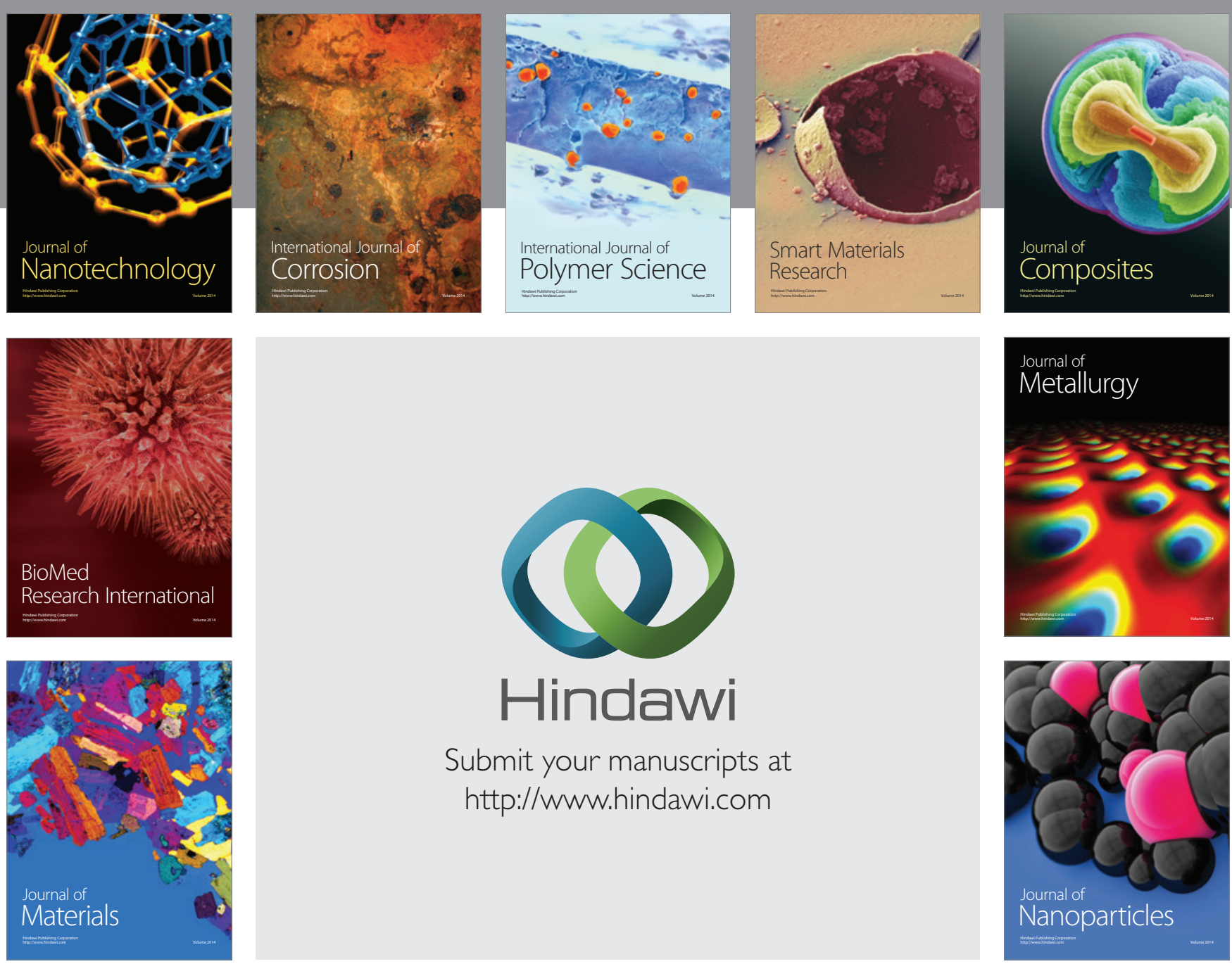

Submit your manuscripts at http://www.hindawi.com
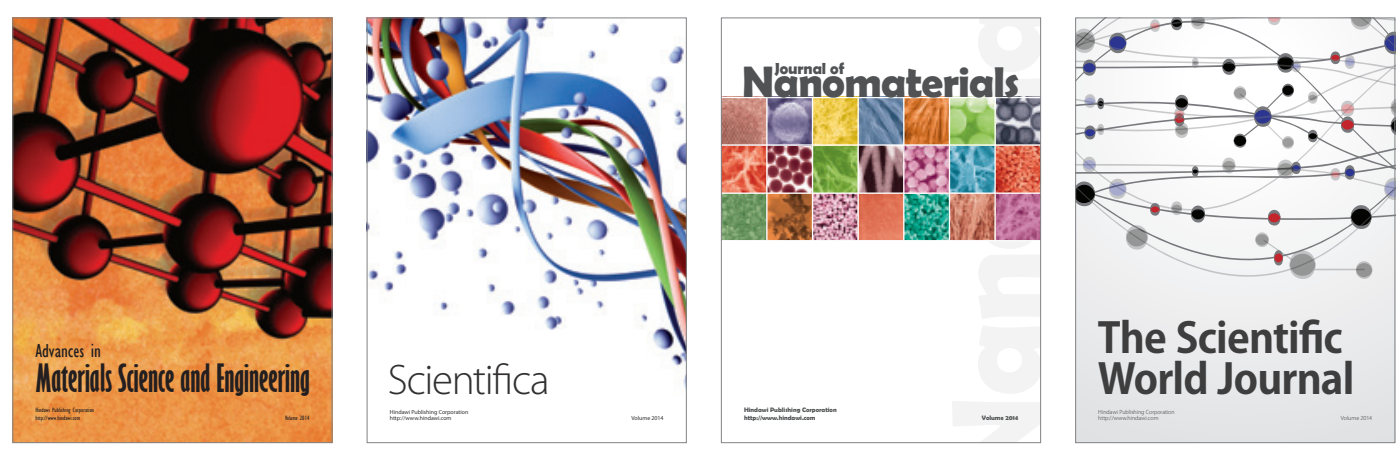

\section{The Scientific World Journal}
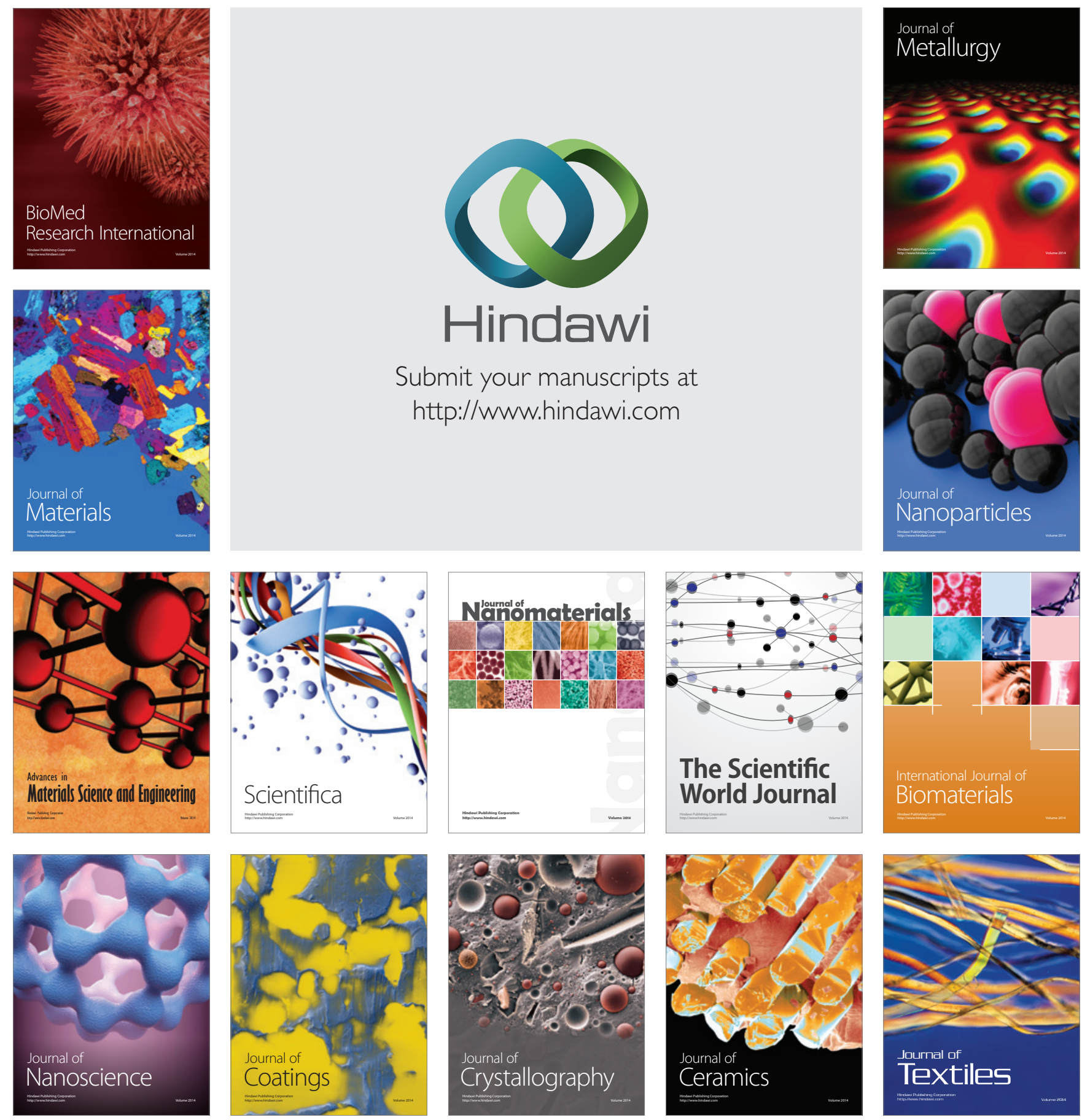